\title{
ON CLOSE-TO-CONVEX ANALYTIC FUNCTIONS
}

\author{
BY \\ CH. POMMERENKE
}

\section{Introduction.}

1.1. For $0 \leqq \alpha \leqq 1$, let $\Omega_{\alpha}$ denote the family of functions $f(z)$ analytic in $|z|<1$ for which there exists a convex function $h(z)$ such that $\left({ }^{1}\right)$

$$
\left|\arg f^{\prime}(z) / h^{\prime}(z)\right| \leqq \frac{\alpha \pi}{2} \quad(|z|<1) .
$$

Here a function is called convex if it maps the unit disk conformally onto a convex region. These classes were introduced by Kaplan [7] and later studied by Reade [19]. In particular, $\boldsymbol{R}_{0}$ is the family of convex functions and $\boldsymbol{R}_{1}$ the family of close-to-convex functions.

Kaplan [7] proved that all close-to-convex functions are univalent, and that an analytic function $f(z)$ with $f^{\prime}(z) \neq 0$ belongs to $\Omega_{\alpha}$ if and only if

$$
\int_{\theta_{1}}^{\theta_{2}} d \arg \left[e^{i \theta} f^{\prime}\left(r e^{i \theta}\right)\right] \geqq-\alpha \pi \quad\left(\theta_{1}<\theta_{2}<\theta_{1}+2 \pi, 0 \leqq r<1\right) .
$$

Later, Lewandowski [8; 9] (see also [2]) gave the following completely geometric characterisation: An analytic univalent function $f(z)$ is close-toconvex in $|z|<1$ if and only if the complement $E$ of the image-region $F=\{f(z):|z|<1\}$ is the union of rays that are disjoint (except that the origin of one ray may lie on another one of the rays).

Similarly, it is possible to prove the following characterisation of the classes $\Omega_{\alpha}$ : An analytic univalent function $f(z)$ belongs to $\Omega_{\alpha}$ if and only if $E$ is the union of rays that are disjoint (except that the origin of one ray may lie on another one of the rays) such that for every ray the sector of angle $(1-\alpha) \pi$ whose bisector is the given ray lies also in $E$.

The most interesting classes of close-to-convex functions are formed by the starlike functions and the functions convex in one direction. A function $f(z)$ with $f(0)=0$ is called starlike if the image region $F$ is starlike with respect to the origin. A necessary and sufficient condition is

$$
\operatorname{Re}\left[z f^{\prime}(z) / f(z)\right]>0 \quad(|z|<1) .
$$

Received by the editors August 22, 1963.

( ${ }^{1}$ If $\alpha>0$ we may replace $\leqq$ by $<$ in (1.1) because equality for one value $z$ implies $f^{\prime}(z)=$ const. $h^{\prime}(z)$ for all $z$, and this means that $f(z)$ is convex too. 
For an elegant proof see [21]. It is well-known that a function $f(z)$ is starlike if and only if it has the form $f(z)=z h^{\prime}(z)$ where $h(z)$ is convex. A function is called convex in one direction [20] if each line of a certain fixed direction intersects $F$ in at most one interval.

1.2. We shall first study a coefficient problem. If

$$
f(z)=\sum_{n=1}^{\infty} a_{n} z^{n} \quad(|z|<1)
$$

is univalent in $|z|<1$ and $M(r)=\max _{|z|=r}|f(z)|$ then

$$
M(r)=O\left((1-r)^{-\lambda}\right) \text { implies } a_{n}=O\left(n^{\lambda-1}\right)
$$

provided that $\lambda>1 / 2[6$, p. 46]. On the other hand, Littlewood has given an example of a bounded univalent function for which $a_{n} \neq O\left(n^{\sigma-1}\right)$ for some positive $\sigma$. Thus the implication (1.3) breaks down for small $\lambda$.

The author conjectures that (1.3) is true for all $\lambda \geqq 0$ if $f(z)$ is close-toconvex. For starlike functions, it is known that (1.3) holds for $\lambda \geqq 0$ [4; 14]. It will be proved for two further special cases:

Theorem 1. Let $f(z)=\sum_{n=1}^{\infty} a_{n} z^{n}$ belong to $\Re_{\alpha}$ with $0 \leqq \alpha<1$ or let $f(z)$ be convex in one direction. If

$$
M(r)=\max _{|z|=r}|f(z)| \leqq \frac{B}{(1-r)^{\lambda}} \quad(0 \leqq r<1)
$$

with $\lambda \geqq 0$ then

$$
\left|a_{n}\right| \leqq K B n^{\lambda-1} \quad(n=1,2, \cdots)
$$

where $K$ depends only on $\alpha$ in the first case and $K \leqq 16 / \pi$ in the second case.

Actually, we will prove a more general result in each case. This theorem generalises in part some earlier results of the author [15] on symmetric functions in $\Omega_{\alpha}$.

1.3. Next we shall consider some invariance properties of the families $\Omega_{\alpha}$. Finally the following theorem about the continuity behaviour will be proved:

THEOREM 2. Let $f \in \Omega_{\alpha}$.

(a) If $0 \leqq \alpha<1$ then $f(z)$ is continuous in $|z| \leqq 1$ (if $\infty$ is allowed as a value) and assumes no finite value more than once.

(b) If $\alpha=1$ then

$$
f\left(e^{i \theta}\right)=\lim _{z \rightarrow e^{i \theta}} f(z)
$$

exists as a finite or infinite angular limit for all $\theta$ with at most two exceptions, and two exceptions may really occur. 
Again, this theorem will be contained in more general results.

2. Coefícient problems.

2.1. Theorem 3. Let $0 \leqq \alpha<1, f(z)=a_{1} z+\cdots \in \Omega_{\alpha}$. If $0 \leqq r<1$ then

$$
r \int_{0}^{2 \pi}\left|f^{\prime}\left(r e^{i \theta}\right)\right| d \theta \leqq K(\alpha) \max _{|z|=r}|f(z)|
$$

where $K(\alpha)$ depends only on $\alpha$.

The left side of (2.1) is equal to the length $L(r)$ of $\{f(z):|z|=r\}$. Since trivially $L(r) \geqq 2 M(r)$, inequality (2.1) shows that $L(r)$ and $M(r)$ have essentially the same growth. (For a related result for starlike functions see $[16$, Theorem 2]).

Proof. Let $v(r, \theta)=\arg \left[e^{i \theta} h^{\prime}\left(r e^{i \theta}\right)\right]$. Then we have by $(1.1)$

$$
\left|\arg \left[e^{i \theta} f^{\prime}\left(r e^{i \theta}\right)\right]-v(r, \theta)\right| \leqq \frac{\alpha \pi}{2} .
$$

Let $m=[8 /(1-\alpha)]+1$. For fixed $r<1$ we define $\theta_{k}$ by

$$
v\left(r, \theta_{k}\right)=2 \pi k / m \quad(k=0, \cdots, m-1)
$$

and $\theta_{m}=\theta_{0}+2 \pi$. Since $v(r, \theta)$ increases with $\theta$ we see that

$$
\theta_{0}<\theta_{1}<\cdots<\theta_{m}=\theta_{0}+2 \pi
$$

and that

$$
0 \leqq v(r, \theta)-v\left(r, \theta_{k}\right) \leqq \frac{2 \pi}{m}<(1-\alpha) \frac{\pi}{4}
$$

for $\theta_{k} \leqq \theta \leqq \theta_{k+1}$. Together with (2.2) this gives

$$
\left|\arg \left[e^{i \theta} f^{\prime}\left(r e^{i \theta}\right)\right]-v\left(r, \theta_{k}\right)\right|<(1+\alpha) \frac{\pi}{4}<\frac{\pi}{2}
$$

for $\theta_{k} \leqq \theta \leqq \theta_{k+1}$. Hence

$$
\begin{aligned}
& \cos \left((1+\alpha) \frac{\pi}{4}\right) \cdot r \int_{\theta_{k}}^{\theta_{k+1}}\left|f^{\prime}\left(r e^{i \theta}\right)\right| d \theta \\
& \leqq r \int_{\theta_{k}}^{\theta_{k+1}}\left|f^{\prime}\left(r e^{i \theta}\right)\right| \cos \left[\arg \left(e^{i \theta} f^{\prime}\left(r e^{i \theta}\right)\right)-v\left(r, \theta_{k}\right)\right] d \theta \\
&=\operatorname{Re}\left[e^{-i v\left(r, \theta_{k}\right)} \int_{\theta_{k}}^{\theta_{k+1}} r e^{i \theta} f^{\prime}\left(r e^{i \theta}\right) d \theta\right] \\
&=\operatorname{Re}\left[e^{-i w\left(r, \theta_{k}\right)-i \pi / 2}\left(f\left(r e^{i \theta_{k+1}}\right)-f\left(r e^{i \theta_{k}}\right)\right)\right] \leqq 2 M(r)
\end{aligned}
$$

for $k=0,1, \cdots, m-1$. By summation we obtain 


$$
r \int_{\theta_{0}}^{\theta_{0}+2 \pi}\left|f^{\prime}\left(r e^{i \theta}\right)\right| d \theta \leqq \frac{2 m M(r)}{\cos ((1+\alpha) \pi / 4)} \leqq \frac{18 M(r)}{(1-\alpha) \sin ((1-\alpha) \pi / 4)} .
$$

Proof of Theorem 1, first case. Let $f \in \Omega_{\alpha}(0 \leqq \alpha<1)$. By Theorem 3 we have

$$
\begin{aligned}
n\left|a_{n}\right| r^{n} & =\left|\frac{r}{2 \pi} \int_{0}^{2 \pi} f^{\prime}\left(r e^{i \theta}\right) e^{-i(n-1) \theta} d \theta\right| \\
& \leqq \frac{r}{2 \pi} \int_{0}^{2 \pi}\left|f^{\prime}\left(r e^{i \theta}\right)\right| d \theta \leqq \frac{1}{2 \pi} K(\alpha) M(r)
\end{aligned}
$$

Taking $r=n /(n+1)$ we obtain $n\left|a_{n}\right| \leqq e K(\alpha) M(n /(n+1)) \leqq K_{1}(\alpha) B n^{\lambda}$.

Corollary 1. If $f(z)$ is bounded and belongs to $\Omega_{\alpha}(0 \leqq \alpha<1)$ then

$$
a_{n}=o\left(n^{-1}\right) \text {. }
$$

Proof. As Theorem 3 shows $f(z)$ belongs to the Hardy class $H_{1}$. If we make $r \rightarrow 1-0$ in (2.3) we see that

$$
n\left|a_{n}\right|=\frac{1}{2 \pi}\left|\int_{0}^{2 \pi} f^{\prime}\left(e^{i \theta}\right) e^{-i(n-1) \theta} d \theta\right|
$$

and the integral tends to 0 by the Riemann-Lebesgue lemma.

2.2. The second case of Theorem 1 is contained in the next theorem.

THEOREM 4. Let $f(z)=a_{1} z+\cdots$ be convex in the direction of the imaginary axis and

$$
A(r)=\max _{|z|=r}|\operatorname{Re} f(z)| \leqq B(1-r)^{-\lambda}
$$

with $\lambda \geqq 0$. Then

$$
\left|a_{n}\right| \leqq \frac{16}{\pi} B n^{\lambda-1}
$$

In particular, if $\lambda=0$ then $\left|a_{n}\right| \leqq 4 B / \pi$, and $4 / \pi$ cannot be replaced by a smaller constant.

Proof. If $f(z)$ is convex in the direction of the imaginary axis then for each fixed $r<1$ there are numbers $\theta_{1}$ and $\theta_{2}$ such that $u(r, \theta)=\operatorname{Re} f\left(r e^{i g}\right)$ increases for $\theta_{1}<\theta<\theta_{2}$ and decreases for $\theta_{2}<\theta<\theta_{1}+2 \pi$. Hence

$$
\int_{0}^{2 \pi}\left|\frac{\partial}{\partial \theta} u(r, \theta)\right| d \theta \leqq 2\left(\max _{\theta} u(r, \theta)-\min _{\theta} u(r, \theta)\right) \leqq 4 A(r) .
$$


From the well-known representation [12, vol. I, p.307] we obtain for $n=1,2, \cdots$

$$
a_{n}=\frac{1}{\pi r^{n}} \int_{0}^{2 \pi} u(r, \theta) e^{-i n \theta} d \theta=\frac{1}{\pi i n r^{n}} \int_{0}^{2 \pi}\left(\frac{\partial}{\partial \theta} u(r, \theta)\right) e^{-i n \theta} d \theta .
$$

Therefore (2.4) shows that

$$
\left|a_{n}\right| \leqq \frac{4}{\pi n r^{n}} A(r)
$$

Let $A(r) \leqq B(1-r)^{-\lambda}$. With $r=(n-1) / n(n=2,3, \ldots)$ we obtain from (2.5)

$$
\left|a_{n}\right| \leqq \frac{4}{\pi} B\left(\frac{n}{n-1}\right)^{n} n^{\lambda-1} \leqq \frac{16}{\pi} B n^{\lambda-1}
$$

If $\lambda=0$ we make $r \rightarrow 1-0$ in (2.5) and find $\left|a_{n}\right| \leqq(4 / \pi) B$. This inequality is best possible as is shown by the function

$$
\frac{2 B}{\pi i} \log \frac{1+z}{1-z}=\frac{4 B}{\pi i} \sum_{k=0}^{\infty} \frac{z^{2 k+1}}{2 k+1}
$$

that is convex in the direction of the imaginary axis and satisfies $A(r) \leqq B$.

3. Invariance properties.

3.1. Lemma 1. If $f(z)$ is analytic, $h(z)$ is convex in $|z|<1$ and

$$
\left|\arg f^{\prime}(z) / h^{\prime}(z)\right| \leqq \frac{\alpha \pi}{2} \quad(0 \leqq \alpha \leqq 1)
$$

then

$$
\left|\arg \frac{f\left(z_{2}\right)-f\left(z_{1}\right)}{h\left(z_{2}\right)-h\left(z_{1}\right)}\right| \leqq \frac{\alpha \pi}{2} \quad\left(\left|z_{1}\right|<1,\left|z_{2}\right|<1\right) .
$$

Proof. Let $\chi(w)$ be the inverse function of $w=h(z)$. Then $\chi(w)$ is analytic in the convex region $\{h(z):|z|<1\}$. Let $g(z)=f(\chi(w))$. With $w_{j}=h\left(z_{j}\right)$ $(j=1,2)$ we can write

$$
\frac{f\left(z_{2}\right)-f\left(z_{1}\right)}{h\left(z_{2}\right)-h\left(z_{1}\right)}=\frac{g\left(w_{2}\right)-g\left(w_{1}\right)}{w_{2}-w_{1}}=\int_{0}^{1} g^{\prime}\left(w_{1}+\left(w_{2}-w_{1}\right) t\right) d t .
$$

Since $s=g^{\prime}(w)=f^{\prime}(\chi(w)) / h^{\prime}(\chi(w))$ lies in the convex sector $\{|\arg s| \leqq \alpha \pi / 2\}$ the same is true of its integral mean value (3.1).

TheOREM 5. Let $\left|z_{1}\right| \leqq 1,\left|z_{2}\right| \leqq 1$. If

$$
f(z)=a_{1} z+\cdots+a_{n} z^{n}+\cdots \in \Omega_{\alpha} \quad(0 \leqq \alpha \leqq 1)
$$

then also 


$$
\int_{0}^{z} \frac{f\left(z_{2} \zeta\right)-f\left(z_{1} \zeta\right)}{z_{2} \zeta-z_{1} \zeta} d \zeta=a_{1} z+\cdots+\frac{z_{2}^{n}-z_{1}^{n}}{n\left(z_{2}-z_{1}\right)} a_{n} z^{n}+\cdots \in \Omega_{\alpha} .
$$

For the special case $z_{1}=0, z_{2}=1$, this was proved by Sakaguchi [22]. Proof. Let $h(z)$ be the convex function of $(1.1)$. Since $z h^{\prime}(z)$ is starlike, inequality (1.2) shows that

$$
\operatorname{Re}\left[\frac{d}{d z}\left(z h^{\prime}(z)\right) / h^{\prime}(z)\right]>0 .
$$

Applying Lemma 1 with $f(z)=z h^{\prime}(z), \alpha=1$ and with $z_{j} z$ instead of $z_{j}$ we therefore obtain

$$
\operatorname{Re} \frac{z_{2} z h^{\prime}\left(z_{2} z\right)-z_{1} z h^{\prime}\left(z_{1} z\right)}{h\left(z_{2} z\right)-h\left(z_{1} z\right)}>0
$$

Let

$$
h_{*}(z)=\int_{0}^{z} \frac{h\left(z_{2} \zeta\right)-h\left(z_{1} \zeta\right)}{z_{2} \zeta-z_{1} \zeta} d \zeta
$$

Then

$$
z \frac{d}{d z}\left(z h_{*}^{\prime}(z)\right) /\left(z h_{*}^{\prime}(z)\right)=\frac{z_{2} z h^{\prime}\left(z_{2} z\right)-z_{1} z h^{\prime}\left(z_{1} z\right)}{h\left(z_{2} z\right)-h\left(z_{1} z\right)}
$$

Hence $h_{*}(z)$ is convex by (3.3). If $f_{*}(z)$ denotes the function defined by (3.2) then Lemma 1 shows that

Therefore $f_{*} \in \Omega_{\alpha}$.

$$
\left|\arg \frac{f_{*}^{\prime}(z)}{h_{*}^{\prime}(z)}\right|=\left|\arg \frac{f\left(z_{2} z\right)-f\left(z_{1} z\right)}{h\left(z_{2} z\right)-h\left(z_{1} z\right)}\right| \leqq \frac{\alpha \pi}{2} .
$$

Corollary 2. If $f(z)=z+\cdots \in \Re_{\alpha}(0 \leqq \alpha \leqq 1)$ then for $\left|z_{1}\right|<1,\left|z_{2}\right|<1$,

$$
\left|\arg \frac{f\left(z_{2}\right)-f\left(z_{1}\right)}{z_{2}-z_{1}}\right|<(1+\alpha) \pi
$$

and the constant on the right side is best possible.

For $z_{1}=z_{2}=z$, inequality (3.4) reduces to $\left|\arg f^{\prime}(z)\right|<(1+\alpha) \pi$, and the constant will be shown to be best possible. In the case $\alpha=1$ this result is contained in the work of Biernacki [3]; for $\alpha=0$ it was proved by Bieberbach [1].

In contrast to the best possible inequality $\left|\arg f^{\prime}(z)\right|<2 \pi$ for analytic close-to-convex functions $f(z)=z+\cdots$ the author has shown [13, Theorem 7] that $\left|\arg \phi^{\prime}(\zeta)\right|<3 \pi / 2$ for meromorphic close-to-convex functions 


$$
\phi(\zeta)=\zeta+b_{0}+b_{1} \zeta^{-1}+\cdots \quad(|\zeta|>1)
$$

Proof. By (1.1) we can write

$$
f^{\prime}(z)=h^{\prime}(z) g(z)
$$

where $h(z)=e^{i \gamma} z+\ldots$ is a convex function and $g(z)=e^{i \gamma}+\ldots$ is a function satisfying $|\arg g(z)| \leqq \alpha \pi / 2$. Therefore $|\gamma|=|\arg g(0)| \leqq \alpha \pi / 2$. Also, $e^{-i \gamma} h(z)=z+\cdots$ is convex and therefore $\left|\arg e^{-i \gamma} h^{\prime}(z)\right|<\pi[1]$. Hence (3.5) shows that

$$
\begin{aligned}
\left|\arg f^{\prime}(z)\right| & \leqq|\gamma|+\left|\arg e^{-i \gamma} h^{\prime}(z)\right|+|\arg g(z)| \\
& <\frac{\alpha \pi}{2}+\pi+\frac{\alpha \pi}{2}=(1+\alpha) \pi .
\end{aligned}
$$

Applying this inequality to the function defined by (3.2) we immediately obtain (3.4).

The functions given by

$$
f^{\prime}(z)=e^{i \tau \alpha}\left(1-e^{i t} z\right)^{-2}\left(e^{-i \tau}+e^{i \tau} z\right)^{\alpha}(1-z)^{-\alpha}
$$

with real $t$ and $-\pi / 2<\tau<\pi / 2$ belong to $\Re_{\alpha}$, and one easily sees that $\left|\arg f^{\prime}(z)\right|$ comes arbitrarily near to $(1+\alpha) \pi$ for suitable $t, \tau$ and $z$.

3.2. A family of functions $f(z)=z+\cdots$ that are analytic in $|z|<1$ and satisfy $f^{\prime}(z) \neq 0$ is called linear-invariant [17] if for every linear transformation $\phi$ of the unit disk onto itself together with $f(z)$ the function

$$
\Lambda_{\phi}[f(z)]=\frac{f(\phi(z))-f(\phi(0))}{\phi^{\prime}(0) f^{\prime}(\phi(0))}=z+\cdots
$$

belongs to the family. The order of a linear-invariant family is defined as $\sup (1 / 2)\left|f^{\prime \prime}(0)\right|$ taken over all functions $f$ in the family. Let $\AA_{\alpha}^{0}$ denote the normalised family consisting of the functions $f(z)=z+\cdots \in \Omega_{\alpha}$.

Lemma 2. The family $\Omega_{\alpha}^{0}(0 \leqq \alpha \leqq 1)$ is linear-invariant and has the order $1+\alpha$.

This result is essentially due to Reade [19]. In order to prove that $\Lambda_{\phi}[f] \in \Re_{\alpha}$ for $f \in \Re_{\alpha}$ one considers the convex function

$$
[h(\phi(z))-h(\phi(0))] /\left[\phi^{\prime}(0) f^{\prime}(\phi(0))\right] .
$$

The lemma implies $\left[17\right.$, Theorem 2.5] that the radius of convexity of $\Omega_{\alpha}$ is equal to $1+\alpha-\left(2 \alpha+\alpha^{2}\right)^{1 / 2}$.

Finally, there is another easily proved invariance property of $\Omega_{\alpha}$ : If $f_{0} \in \Omega_{\alpha}, f_{1} \in \Re_{\alpha}$ then

$$
f_{\lambda}(z)=\int_{0}^{z} f_{0}^{\prime}(\zeta)^{1-\lambda} f_{1}^{\prime}(\zeta)^{\lambda} d \zeta \in \Omega_{\alpha} .
$$




\section{The continuity behaviour.}

4.1. We shall first consider the case $0 \leqq \alpha<1$. If $F$ is a region in the plane and $w_{1} \in F, w_{2} \in F$ let

$$
l\left(w_{1}, w_{2}\right)=l_{F}\left(w_{1}, w_{2}\right)=\inf _{V} \int_{V}|d w|
$$

where $V$ runs through all rectifiable curves lying in $F$ that connect $w_{1}$ and $w_{2}$.

Theorem 6. Let $0 \leqq \alpha<1$ and $f(z)=z+\cdots \in \Re_{\alpha}, \quad F=\{f(z):|z|<1\}$. If $\left|z_{1}\right|<1,\left|z_{2}\right|<1$ then

$$
\kappa(\alpha)\left|z_{1}-z_{2}\right|^{1+\alpha} \leqq l_{F}\left(f\left(z_{1}\right), f\left(z_{2}\right)\right) \leqq K(\alpha)\left|f\left(z_{1}\right)-f\left(z_{2}\right)\right|
$$

where $\kappa(\alpha)>0$ and $K(\alpha)$ depend only on $\alpha$.

The right inequality (4.1) implies that $f(z)$ is continuous and univalent in $|z| \leqq 1$ except that $\infty$ may be assumed a finite number of times $[18$, Theorem 3.9]. Therefore the assertion of Theorem 2(a) follows from Theorem 6.

Proof. Let $\left|\arg f^{\prime}(z) / h^{\prime}(z)\right| \leqq \alpha \pi / 2$ where $h(z)$ is convex. Let $\chi(w)$ denote the inverse function of $w=h(z)$. For given $z_{0}$ with $\left|z_{0}\right|<1$ let $w_{0}=h\left(z_{0}\right)$ and let $C_{0}$ be the image of the segment $\left[0, w_{0}\right]$ under $z=\chi(w)$. The function $g(w)=f(\chi(w))$ satisfies $\left|\arg g^{\prime}(w)\right| \leqq \alpha \pi / 2$. Therefore

$$
\begin{aligned}
\cos \frac{\alpha \pi}{2} \cdot \int_{C_{0}}\left|f^{\prime}(z)\right| d z \mid & =\left|w_{0}\right| \cos \frac{\alpha \pi}{2} \cdot \int_{0}^{1}\left|g^{\prime}\left(w_{0} t\right)\right| d t \\
& \leqq\left|w_{0}\right| \int_{0}^{1}\left|g^{\prime}\left(w_{0} t\right)\right| \cos \left[\arg g^{\prime}\left(w_{0} t\right)\right] d t \\
& \leqq\left|w_{0}\right| \operatorname{Re}\left[w_{0}^{-1} g\left(w_{0}\right)\right] \leqq\left|g\left(w_{0}\right)\right|=\left|f\left(z_{0}\right)\right|
\end{aligned}
$$

It follows that

$$
l\left(0, f\left(z_{0}\right)\right) \leqq\left(\cos \frac{\alpha \pi}{2}\right)^{-1}\left|f\left(z_{0}\right)\right|,
$$

and the right inequality (4.1) follows from Lemma 2.

The normalised family $\hat{\Omega}_{\alpha}^{0}$ is linear-invariant and of order $1+\alpha$. Since all close-to-convex functions are univalent the family $\Omega_{\alpha}^{0}$ is also of bounded characteristic (beschränktartig). Therefore [17, Theorem 2.9]

$$
l\left(f\left(z_{1}\right), f\left(z_{2}\right)\right) \geqq \kappa(\alpha)\left|z_{1}-z_{2}\right|^{1+\alpha} .
$$

CoRollary 3. If $f(z)=a_{1} z+\cdots \in \Omega_{\alpha}(0 \leqq \alpha<1)$ then 


$$
\int_{0}^{r}\left|f^{\prime}\left(\rho e^{i \theta}\right)\right| d \rho \leqq K_{1}(\alpha)\left|f\left(r e^{i \theta}\right)\right| \quad(0 \leqq r<1,0 \leqq \theta \leqq 2 \pi) .
$$

This assertion is a consequence of the right inequality (4.1) and the following theorem of Gehring and Hayman [5] (see also [17, Theorem 2.7]):

There exists an absolute constant $K_{0}$ such that for all univalent functions $f(z)$ with $F=\{f(z):|z|<1\}$

$$
\int_{0}^{r}\left|f^{\prime}\left(\rho e^{i \theta}\right)\right| d \rho \leqq K_{0} l_{F}\left(f(0), f\left(r e^{i \theta}\right)\right)
$$

4.2. We shall study now the case $\alpha=1$. We need the following lemma.

Lemma 3. Let $h(z)$ be convex in $|z|<1$. Then

$$
v(\theta)=\lim _{r \rightarrow 1} \arg \left[e^{i \theta} h^{\prime}\left(r e^{i \theta}\right)\right]
$$

exists for all $\theta$ and is a monotone increasing function. If $h\left(e^{i \theta}\right)$ is finite then

$$
\left|\arg \left[h\left(e^{i \theta}\right)-h(z)\right]-v(\theta)\right|<\frac{\pi}{2} \quad(|z|<1) .
$$

Proof. The function $z h^{\prime}(z)$ is starlike. Therefore the limit (4.2) exists and defines a monotone function [14, Lemma 1]. If $|z|<1$ then

$$
h((\zeta+z) /(1+\bar{z} \zeta))-h(z)
$$

is starlike (with respect to 0 ) as a function of $\zeta$. Therefore by (1.2)

$$
\left|\arg \left[\frac{\left(1-|z|^{2}\right) \zeta}{(1+\bar{z} \zeta)^{2}} h^{\prime}\left(\frac{\zeta+z}{1+\bar{z} \zeta}\right)\right]-\arg \left[h\left(\frac{\zeta+z}{1+\bar{z} \zeta}\right)-h(z)\right]\right|<\frac{\pi}{2} .
$$

When we choose $\zeta$ such that $(\zeta+z) /(1+\bar{z} \zeta)=r e^{i \theta}$ and let $r \rightarrow 1-0$ we see that

$$
\left|v(\theta)-\arg \left[h\left(e^{i \theta}\right)-h(z)\right]\right| \leqq \frac{\pi}{2}
$$

for $|z|<1$. Since $h(z)$ is not constant equality cannot hold.

Theorem 7. Let $f(z)$ be close-to-convex and let $h(z)$ be a fixed convex function such that $\operatorname{Re}\left[f^{\prime}(z) / h^{\prime}(z)\right]>0$. Let $v(\theta)$ be defined by (4.2). Then

$$
f\left(e^{i \theta}\right)=\lim _{z \rightarrow e^{i \theta}} f(z)
$$

exists as a finite or infinite angular limit for all $\theta$ for which $v(\theta+0)-v(\theta-0) \neq \pi$.

The proof will be based on the theory of prime ends. If $f(z)$ is univalent then there is a one-to-one correspondence between the points on the unit circle and the prime ends of the image-region $F$. The prime end corresponding to $e^{i \theta}$ will be denoted by $P(\theta)$. A point $\omega$ is called a principal point of $P(\theta)$ if every neighborhood of $\omega$ contains a cross-cut of a chain defining $P(\theta)$. Every prime end has either exactly one principal point (possibly at $\infty$ ) or 
infinitely many principal points. A theorem of Lindelöf [10] states that the angular limit $f\left(e^{i \theta}\right)$ exists if and only if $P(\theta)$ has exactly one principal point.

Proof. (a) Let $\theta$ be such that $h\left(e^{i \theta}\right)$ is finite, and let $\omega$ be a finite point of $P(\theta)$. Applying Lemma 1 to $z$ and a sequence $z_{n}$ with $z_{n} \rightarrow e^{i \theta}, f\left(z_{n}\right) \rightarrow \omega$, we see that

$$
\left|\arg [\omega-f(z)]-\arg \left[h\left(e^{i \theta}\right)-h(z)\right]\right| \leqq \frac{\pi}{2} .
$$

By (4.3) it follows that

$$
|\arg [\omega-f(z)]-v(\theta)|<\pi
$$

for $|z|<1$. Hence the ray

$$
T(\theta, \omega)=\{w: \arg (w-\omega)=v(\theta)\}
$$

lies in the complement $E$ of $F$.

(b) Let now $\theta$ be arbitrary and let $\omega$ be a finite principal point of $P(\theta)$. Then there is a chain of cross-cuts $Q_{k}$ of $F$ that defines $P(\theta)$ such that $Q_{k}$ converges to $\omega$. If $\omega_{k}^{ \pm} \in P\left(\theta_{k}^{ \pm}\right)$are the endpoints of $Q_{k}$ we may assume that $h\left(e^{i \theta_{k}^{ \pm}}\right)$is finite. We have $v\left(\theta_{k}^{ \pm}\right) \rightarrow v(\theta \pm 0)$ and $\omega_{k}^{ \pm} \rightarrow \omega$. Therefore (4.4) shows that $T_{k}^{ \pm}=T\left(\theta_{k}^{ \pm}, \omega_{k}^{ \pm}\right)$converges to $T(\theta \pm 0, \omega)=\{w: \arg (w-\omega)=v(\theta \pm 0)\}$. Because $T_{k}^{ \pm} \subset E$ by part (a), the Jordan curve $J_{k}=T_{k}^{-} \cup Q_{k} \cup T_{k}^{+}$separates the plane into two regions one of which contains $f(0)$, and the closure $A_{k}$ of the other region contains $\omega^{\prime}$ where $\omega^{\prime}$ is any point of $P(\theta)$. Since $Q_{k}$ converges to $\omega$ the Jordan curve $J_{k}$ converges to $J(\theta, \omega)=T(\theta+0, \omega) \cup T(\theta-0, \omega)$. Since $T_{k}^{ \pm} \subset E$ and $E$ is closed it follows that $J(\theta, \omega) \subset E$. The closed sector determined by $J(\theta, \omega)$ that does not contain $f(0)$ must contain $\omega^{\prime}$ because $\omega^{\prime} \in A_{k}$. As $\omega^{\prime}$ is a boundary point of $F$ this implies $\omega^{\prime} \in J(\theta, \omega)$.

(c) Suppose that the angular limit $f\left(e^{i \theta}\right)$ does not exist. Then $P(\theta)$ has infinitely many principal points. Hence there are at least two different finite principal points $\omega$ and $\omega^{\prime}$. By the result of part (b), we have $\omega^{\prime} \in J(\theta, \omega)$ and also $\omega \in J\left(J, \omega^{\prime}\right)$. Since $J(\theta, \omega)$ and $J\left(\theta, \omega^{\prime}\right)$ are identical except for a translation it follows that the angle $v(\theta+0)-v(\theta-0)$ of $J(\theta, \omega)$ has to be equal to $\pi$.

Proof of Theorem 2(b). The function $v(\theta)$ increases monotonely by $2 \pi$ in $0 \leqq \theta \leqq 2 \pi$. Hence $v(\theta)$ can have at most two jumps of height $\pi$. Therefore $f\left(e^{i \theta}\right)$ exists for all $\theta$ with at most two exceptions.

We shall now give an example where two exceptions occur. Let

$$
\begin{array}{r}
F=\{w=x+i y:|x|<2,|y|<2\} \backslash \bigcup_{k=1}^{\infty}\left\{|x|=2-\frac{1}{2 k},-1 \leqq y<2\right\} \\
\bigcup_{k=1}^{\infty}\left\{|x|=2-\frac{1}{2 k+1},-2<y \leqq 1\right\} .
\end{array}
$$


Here all points $\{x=2,|y| \leqq 1\}$ and $\{x=-2,|y| \leqq 1\}$ are principal points of one prime end each. The function that maps the unit disk onto $F$ is convex in the direction of the imaginary axis, hence close-to-convex, and there are two values of $\theta$ where the angular limit does not exist.

REMARK. If there are two points where the angular limit does not exist then we see that $v(\theta)$ is constant except for two jumps of height $\pi$. It is not difficult to show that this implies that $f(z)$ is convex in one direction.

\section{REFERENCES}

1. L. Bieberbach, Aufstellung und Beweis des Drehungssatzes für schlichte konforme Abbildungen, Math. Z. 4 (1919), 295-305.

2. A. Bielecki and Z. Lewandowski, Sur une théorème concernant les fonctions univalentes linéairement accessibles de M. Biernacki, Ann. Polon. Math. 12 (1962), 61-63.

3. M. Biernacki, Sur la représentation conforme des domaines linéairement accessibles, Prace Mat. Fiz. 44 (1936), 293-314.

4. J. Clunie and F. R. Keogh, On starlike and convex schlicht functions, J. London Math. Soc. 35 (1960), 229-236.

5. F. W. Gehring and W. K. Hayman, An inequality in the theory of conformal mapping, J. Math. Pures Appl. 41 (1963), 353-361.

6. W. K. Hayman, Multivalent functions, University Press, Cambridge, 1958.

7. W. Kaplan, Close-to-convex schlicht functions, Michigan Math. J. 1 (1952), 169-185.

8. Z. Lewandowski, Sur l'identité de certaines classes de fonctions univalentes. I, Ann. Univ. Mariae Curie-Sky. Sect. A 12 (1958), 131-145.

9. , Sur l'identité de certaines classes de fonctions univalentes. II. Ann. Univ. Mariae Curie-Skł. Sect. A 14 (1960), 19-46.

10. E. Lindelöf, Sur un principe général de l'analyse et ses applications à la représentation conforme, Acta Soc. Sci. Fenn. Nova Ser. A 46 (1920), no. 4 (1915).

11. J. E. Littlewood, On the coefficients of schlicht functions, Quart. J. Math. 9 (1938), 14-20.

12. G. Pólya and G. Szegö, Aufgaben und Lehrsätze aus der Analysis, Springer-Verlag, Berlin, 1925.

13. Ch. Pommerenke, Uber einige Klassen meromorpher schlichter Funktionen, Math. Z. 78 (1962), 263-284.

14. , On starlike and convex functions, J. London Math. Soc. 37 (1962), 209-224.

15. , On the coefficients of close-to-convex functions, Michigan Math. J. 9 (1962), 259-269.

16. On starlike and close-to-convex functions, Proc. London Math. Soc. 13 (1963), 290-304.

17. , Linear-invariante Familien analytischer Funktionen. I, Math. Ann. 155 (1964), 108-154.

18. , Linear-invariante Familien analytischer Funktionen. II, Math. Ann. 156 (1964), 226-262.

19. M. O. Reade, The coefficients of close-to-convex functions, Duke Math. J. 23 (1956), 456-462.

20. M. S. Robertson, On the theory of univalent functions, Ann. of Math. 37 (1936), 374-408. 21. 11 (1961), 315-324.

22. K. Sakaguchi, On a certain univalent mapping, J. Math. Soc. Japan 11 (1959), 72-75.

\section{UNIVERSITÄT GöTTINGEN,} Göttingen, Germany 